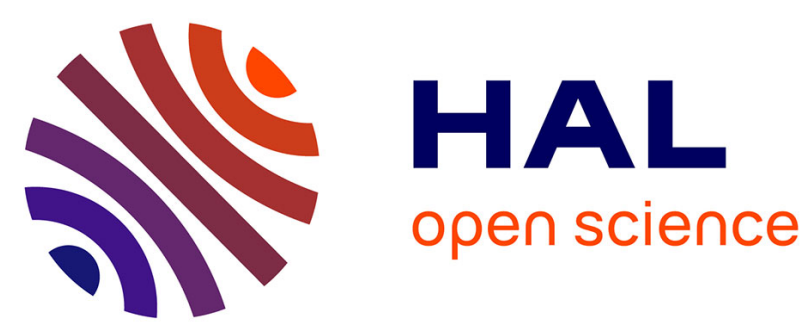

\title{
MODÉLISATION DE LA DIFFRACTION ACOUSTIQUE D'UNE ONDE INCIDENTE PLANE PAR UNE STRUCTURE ÉLASTIQUE À L'AIDE D'UNE MÉTHODE MIXTE ÉLÉMENTS FINIS-ÉQUATIONS INTÉGRALES
}

\author{
A. Lavie, Bertrand Dubus
}

\section{To cite this version:}

A. Lavie, Bertrand Dubus. MODÉLISATION DE LA DIFFRACTION ACOUSTIQUE D'UNE ONDE INCIDENTE PLANE PAR UNE STRUCTURE ÉLASTIQUE À L'AIDE D'UNE MÉTHODE MIXTE ÉLÉMENTS FINIS-ÉQUATIONS INTÉGRALES. Journal de Physique IV Proceedings, 1992, 02 (C1), pp.C1-957-C1-960. 10.1051/jp4:19921209 • jpa-00251175

HAL Id: jpa-00251175 https://hal.science/jpa-00251175

Submitted on 1 Jan 1992

HAL is a multi-disciplinary open access archive for the deposit and dissemination of scientific research documents, whether they are published or not. The documents may come from teaching and research institutions in France or abroad, or from public or private research centers.
L'archive ouverte pluridisciplinaire HAL, est destinée au dépôt et à la diffusion de documents scientifiques de niveau recherche, publiés ou non, émanant des établissements d'enseignement et de recherche français ou étrangers, des laboratoires publics ou privés. 


\title{
MODÉlISATION DE LA DIFFRACTION ACOUSTIQUE D'UNE ONDE INCIDENTE PLANE PAR UNE STRUCTURE ÉLASTIQUE À L'AIDE D'UNE MÉTHODE MIXTE ÉLÉMENTS FINIS-ÉQUATIONS INTÉGRALES
}

\author{
A. LAVIE et B. DUBUS
}

Laboratoire d'Acoustique, URA 253 CNRS, Institut Supérieur d'Electronique du Nord, 41 Boulevard Vauban, F-59046 Lille cedex, France

\begin{abstract}
Résumê - Cet article présente une solution au problème de diffusion acoustique par une structure élastique soumise à une onde incidente plane. Cette solution consiste à effectuer un couplage entre le code ATILA basé sur la mëthode des éléments finis et le code EQI basé sur la méthode des équations intégrales. Le premier permet de modéliser le comportement vibratoire de la structure et le second la propagation des ondes dans le fluide d'extension infinie contenant la structure. Ia théorie du couplage est décrite et appliquée avec succès à la diffusion par une sphère creuge élastique et par un corps cylindrique terminé par deux hémisphères.
\end{abstract}

\begin{abstract}
This article deals with the solution of the scattering of a plane acoustic wave by an elastic structure. This solution consists in the coupling a finite element code (ATIIA) and a boundary element code (EQI). The first one enables the modeling of the vibrating structure and the second one the modeling of propagating waves in the infinite fluid medium surrounding the body. The coupling method is described and successfully applied to the scattering by an elastic spherical shell and a cylinder with hemispherical endcaps.
\end{abstract}

\section{I - INTRODUCTION}

Dans le domaine de l'Acoustique sous-marine, il est primordial de savoir décrire le comportement vibratoire d'une structure immergée dans un fluide et celui des ondes acoustiques générées par cette vibration ou génératrices de cette vibration. La géomêtrie complexe des structures concernées implique l'utilisation d'une méthode numérique. Au laboratoire d'Acoustique de l'ISEN, deux codes de calcul ont été développés et permettent de fournir une solution à ce problème : le code ATILA [1] qui est basé sur la méthode des éléments finis et permet la modélisation de structures élastiques et le code EQI [2] qui utilise la méthode des équations intégrales et permet de modéliser la diffusion acoustique dans le fluide d'extension infinie. La prise en compte simultanée de la vibration de la structure et du fluide environnant est obtenue par un couplage entre ces deux méthodes.

Dans un premier temps, la formulation du problème est brièvement décrite : les méthodes des équations intêgrales et des éléments finis sont successivement rappelées et leur couplage est détaillé. Ensuite, deux applications sont présentées. La première porte sur la diffusion $d$ 'une onde incidente plane par une sphère élastique et la seconde sur la diffusion d'une onde incidente plane dirigée suivant l'axe d'une structure composée d'un corps cylindrique et de deux extrémités hémisphériques. 


\section{II - FORMUTATION DU PROBLEME}

II. 1 - Modélisation du milieu fluide

La méthode des équations intégrales, et plus précisément la représentation de Helmholtz extérieure [3], est particulièrement bien adaptée à la modélisation des ondes acoustiques se propageant dans le fluide entourant la structure. Avec une dépendance temporelle des variables suivant $e^{-i \omega t}$, la formulation intégrale est :

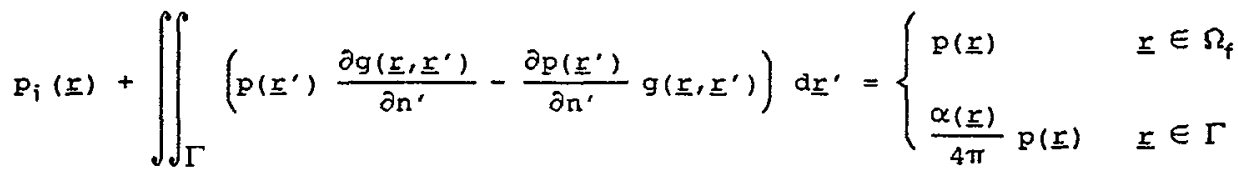

où $p_{i}$ est la pression incidente, $p$ la pression totale, $\underline{r}$ le point d'application de l'equation, $\underline{r}$ le point courant sur la surface $\Gamma, \partial / \partial n^{\prime}$ la dérivée normale par rapport a $\underline{r}^{\prime}$ dirigée positivement vers le fluide $\Omega_{f}, g$ la fonction de Green associée à l'espace infini et $\alpha$ l'angle solide à $\Gamma$ vu de l'exterieur (figure 1). La géométrie, les champs de pression et de sa dérivée normale sont approchés par des fonctions de forme à variation quadratique. La discrétisation aboutit à un système linéaire d’équations :

$$
[A]\{p\}=\{B]\{\partial p / \partial n\}-\left\{p_{\mathbf{i}}\right\}
$$

où [A] et [B] sont les matrices contenant les coefficients d'influence entre tous les noeuds du maillage, $\{p\},\{\partial p / \partial n\}$ et $\left\{p_{j}\right\}$ les vecteurs des pressions totales, de leurs dérivées normales et des pressions incidentes aux noeuds du maillage.

II.2 - Modélisation de la structure élastique

La mêthode des éléments finis permet la modélisation du comportement vibratoire d'une structure élastique de forme quelconque. La géométrie et les champs de déplacement et de pression sont approchés par des fonctions d'interpolation du même type que celles citées précédemment. Après discrétisation, le système linéaire classique est :

$$
\left([K]-\omega^{2}[M]\right)\{U\}=[L]\{p\}
$$

où $\omega$ est la pulsation, [K] et [M] les matrices de rigidité et de masse de la structure, [L\} la matrice de connectivité fluide-structure, \{U\} le vecteur des déplacements aux noeuds de $\Omega_{\mathrm{s}}$ et $\Gamma$ et $\{p\}$ celui des pressions nodales sur $\Gamma$.

\section{II.3 - Couplage fluide-structure}

Le traitement du problème complet fluide-structure repose sur la combinaison des equations (3) et (2) après identification du vecteur $\{p\}$ :

$$
\{p\}=[A]^{-1}[B]\{\partial p / \partial n\}-[A]^{-1}\left\{p_{i}\right\}
$$

La continuité des vitesses à l'interface fluide-structure donne :

$$
\frac{\partial p(\underline{x})}{\partial n}=\rho \omega^{2} u_{n}(\underline{x}) \quad \underline{r} \in \Gamma
$$

où $\rho$ est la masse volumique du fluide et $u_{n}$ le déplacement normal. Pour Éviter le problème de duplicité des normales aux points anguleux du maillage, il est nécessaire de décomposer le déplacement normal suivant les trois composantes du repêre cartêsien global. $\left[B_{p}\right]$ étant la matrice projetée suivant $x, y$ et $z$, il vient :

$$
\left(\left([K]-\omega^{2}[M]\right)-p \omega^{2}[L][A]^{-1}\left[B_{p}\right]\right)\{U\}=-[L][A]^{-1}\left\{p_{i}\right\}
$$


A partir des pressions nodales incidentes, la résolution de ce système fournit les valeurs du déplacement dans $\Omega_{\mathrm{s}}$ et en particulier sur $\Gamma$. La dernière étape consiste alors à calculer la pression sur la surface en fonction des déplacements sur $\Gamma$ par l'intermédiaire de la relation (4). Ensuite, le calcul de ia pression en tout point du fluide ne présente aucune difficulté.

Remarque : si la fréquence de travail coïncide avec une fréquence irrégulière, une technique de surdétermination par des équations de champ nul $[4,5]$ ne modifierait que le système linéaire (2) et la même procédure de couplage serait employée.

\section{III - APPLICATIONS}

\section{III.1 - Diffusion par une sphère élastique}

Le premier corps élastique étudié est une coque sphérique remplie d'air. Le matériau constitutif est un aluminium de module d'Young $\mathrm{E}=7,14.10^{10} \mathrm{~Pa}$, de coefficient de poisson $\nu=0,344$ et de masse volumique $\rho=2780 \mathrm{~kg} / \mathrm{m}^{3}$. L'épaisseur est telle que $b / a=0,9$ où $b$ est le rayon interne et a le rayon externe. Le maillage est suffisamment dense pour assurer la convergence des calculs jusqu'à ka' = 6 . Deux résultats sont présentés : le spectre de rétrodiffusion (figure 2) et 1 'index de réflexion pour $\mathrm{ka}=1,73$ (figure 3), valeur correspondant au premier maximum sur la courbe de rétrodiffusion. La vitesse du son dans le fluide est $c=1490 \mathrm{~m} / \mathrm{s}$. Ces résultats sont comparés avec succès à ceux d'un modèle analytique [6]. Ce premier test constitue une première validation du couplage. Le point calculé a ka = 3,12 est légèrement décalé par rapport à la courbe analytique, probablement à cause de l'influence du voisinage immédiat de la première fréquence irrégulière ka $=\pi$.

III. 2 - Diffusion par une structure cylindrique avec extrémités hémisphériques

Le second corps étudié est une structure cylindrique creuse fermée par des extrếmités hémisphêriques telle que b/a $=0,97$ (figure 4). Le matériau est un acier $\left(E=1,97.10^{11} \mathrm{~Pa}, \quad v=0,3, \rho=7900 \mathrm{~kg} / \mathrm{m}^{3}\right)$. Cette structure est soumise à une onde incidente plane suivant son axe 2. Le rapport b/a autorise l'utilisation d'éléments finis de type coque [7]. Avec un maillage suffisamment dense, le calcul est mené pour une fréquence telle que $k a=8,33(c=1470 \mathrm{~m} / \mathrm{s})$. Le test classique de la source ponctuelle valide parfaitement le traitement numérique de la résolution [3]. $L$ 'index de réflexion calculé est comparé à celui obtenu quand la surface est supposée parfaitement rigide (figure 5). Les déplacements obtenus sont tracés sur la figure 6. On observe que la structure vibre en régime forcé suivant son axe avec une longueur d'onde identique à celle de la propagation de l'onde acoustique dans l'eau.

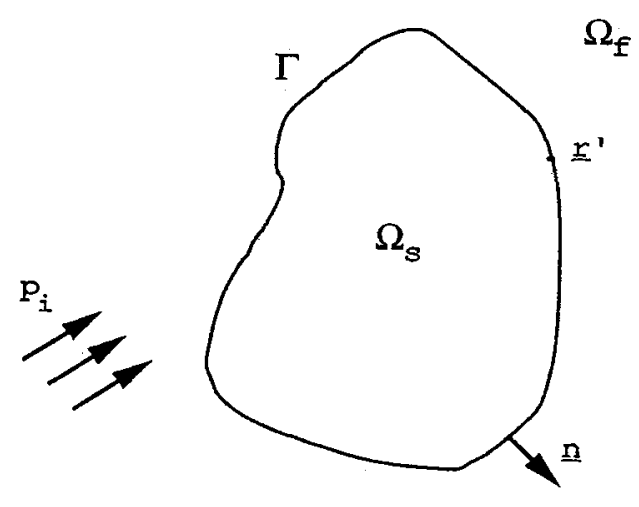

figure 1

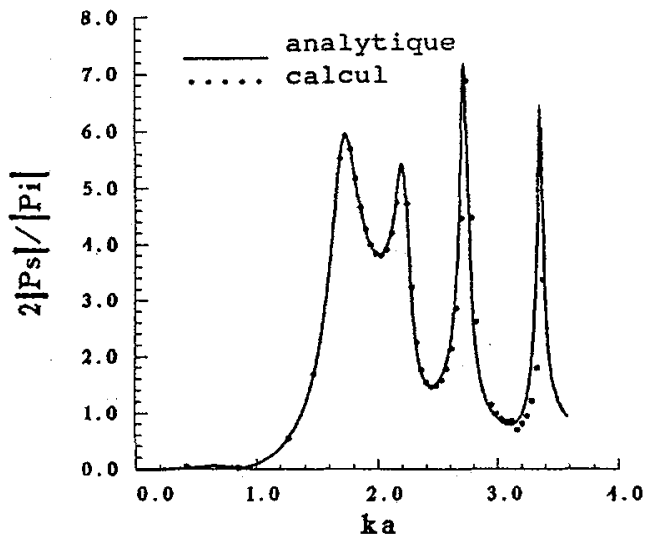

figure 2 


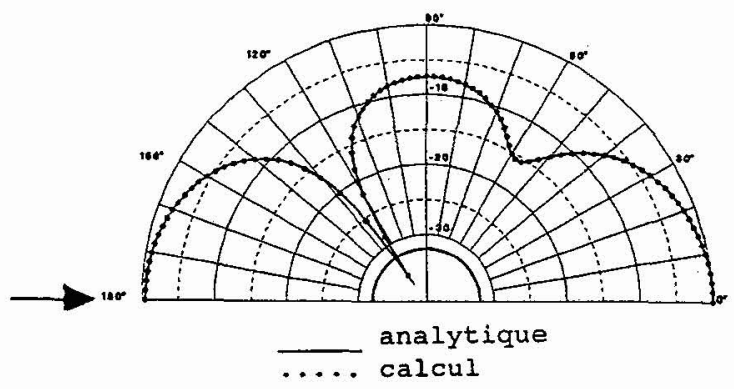

figure 3

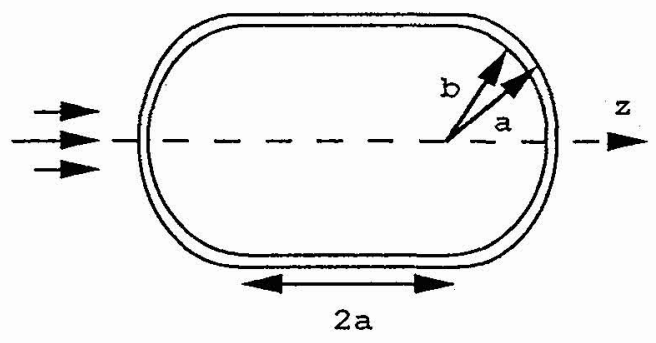

figure 4

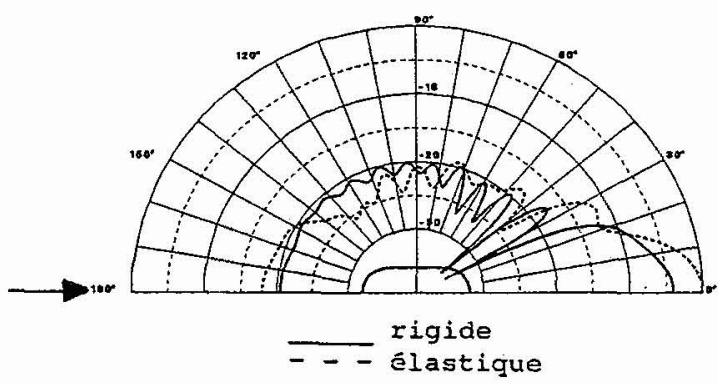

Eigure 5

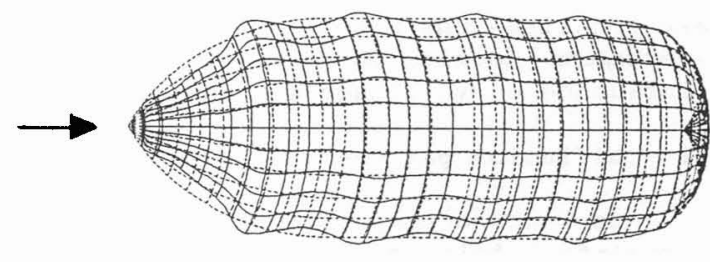

partie réelle déplacement

- - structure au repos

figure 6

\section{IV - CONCLUSIONS}

Ce document commente le couplage entre les méthodes des éléments finis et des équations intégrales par l'intermédiaire des codes ATILA et EQI. Leg résultats obtenus permettent de valider l'outil numérique mis en oeuvre. Les possibilités tridimensionnelles de ces deux codes laissent envisager la modélisation de structures beaucoup plus complexes.

\section{Références}

1. J.-N. DECARPIGNY, "Application de la méthode des éléments finis à 1 'étude de transducteurs piézoélectriques", Thèse de Doctorat d'Etat, Université des sciences et Techniques de Iille (1984).

2. A. LAVIE, "Modélisation du rayonnement ou de la diffraction acoustique par une méthode mixte équations intégrales-champ nul", Thèse de Doctorat, Université des Sciences et Techniques de Lille (1989).

3. H.A. SCHENCK, J. Acoust. Soc. Am., 44, 41-58 (1967).

4. D.S. Jones, Q. J. Mech. Appl. Math., XXII, 129-142 (1973).

5. B. STUPFel et al, J. Acoust. Soc. Am., 83, 927-941 (1988).

6. R. HICKLING, J. Acoust. Soc. Am., 36, 1124-1137 (1964).

7. SOHRABUDDIN AHMAD et al, Int. J. Num. Met. Engng, 2, 419-451 (1970). 